\title{
U(3) and Pseudo-U(3) Symmetry of the Relativistic Harmonic Oscillator
}

\author{
Joseph N. Ginocchio \\ MS B283, Theoretical Division, Los Alamos National Laboratory, \\ Los Alamos, New Mexico 87545, USA
}

(Dated: August 31, 2018)

\begin{abstract}
We show that a Dirac Hamiltonian with equal scalar and vector harmonic oscillator potentials has not only a spin symmetry but an U(3) symmetry and that a Dirac Hamiltonian with scalar and vector harmonic oscillator potentials equal in magnitude but opposite in sign has not only a pseudospin symmetry but a pseudo-U(3) symmetry. We derive the generators of the symmetry for each case.

PACS numbers: 21.60.-n, 21.10.-k, 02.20.-a
\end{abstract}

Keywords: Symmetry, Dirac Hamiltonian, Relativistic mean field theory, Spin, Pseudospin, $\mathrm{SU}(3)$, pseudo-SU(3) 
As is well-known, the non-relativistic spherical harmonic oscillator has degeneracies in ad 2 dtion to those due to rotational invariance. The energy spectrum depends only on the total harmonic oscillator quantum number $N, N=2 n+\ell$, where $\mathrm{n}$ is the radial quantum number and $\ell$ is the orbital angular momentum. Hence the states with $\ell=N, N-2, \ldots 0$ or 1 have the same energy. These degeneracies are produced by an U(3) symmetry [1]. This U(3) symmetry has been influential in connecting the shell model with collective motion [2]. Also the energy does not depend on the orientation of the spin and hence the non-relativistic harmonic oscillator has a spin symmetry as well.

Since relativistic models of nuclei are now so prevalent [3], we can ask if $U(3)$ symmetry resides in the relativistic harmonic oscillator. Indeed the Dirac Hamiltonian, $H$, for which the scalar, $V_{S}(\vec{r})$, and vector, $V_{V}(\vec{r})$, potentials are equal and harmonic has been solved analytically and is invariant under a spin symmetry [4, $[5]$; that is $[\vec{S}, H]=0$ where $\vec{S}$ is given in Eq.(2). Just as for the non-relativistic harmonic oscillator, the spherically symmetric relativistic harmonic oscillator energy spectrum depends only on the total harmonic oscillator quantum number $N$, although the energy spectrum for the relativistic harmonic oscillator spectrum in general does not have a linear dependence on $N$ as does the non-relativistic harmonic oscillator. This suggests that the relativistic harmonic oscillator does have an $\mathrm{U}(3)$ symmetry. If this is the case, the question is: what are the relativistic generators? In this letter we shall show that there is indeed a $\mathrm{U}(3)$ symmetry and we shall derive the generators.

The Dirac Hamiltonian for a spherical harmonic oscillator with spin symmetry is

$$
H=\vec{\alpha} \cdot \vec{p}+\beta M+(1+\beta) V(r),
$$

where $\vec{\alpha}, \beta$ are the Dirac matrices, $\vec{p}$ is the momentum, $M$ is the mass, $V(r)=\frac{M \omega^{2}}{2} r^{2}, \vec{r}$ is 
the radial coordinate, $r$ its magnitude, and the velocity of light is set equal to unity, $\mathrm{c}=13$. The generators for the spin $\mathrm{SU}(2)$ algebra and the orbital angular momentum $\mathrm{SU}(2)$ algebra, $\vec{S}, \vec{L}$, which commute with the Dirac Hamiltonian, $[H, \vec{S}]=[H, \vec{L}]=0$, are given by $[\underline{6}]$

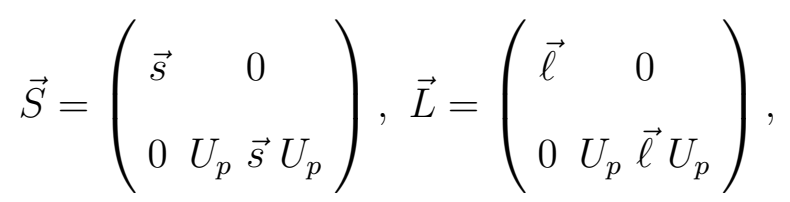

where $\vec{s}=\vec{\sigma} / 2$ are the usual spin generators, $\vec{\sigma}$ the Pauli matrices, $\vec{\ell}=\frac{(\vec{r} \times \vec{p})}{\hbar}$, and $U_{p}=\frac{\overrightarrow{\boldsymbol{\sigma}} \cdot \overrightarrow{\boldsymbol{p}}}{p}$ is the helicity unitary operator introduced in [7].

The non-relativistic $\mathrm{U}(3)$ generators are the orbital angular momentum $\vec{\ell}$, the quadrupole operator $q_{m}=\frac{1}{\hbar M \omega} \sqrt{\frac{3}{2}}\left(M^{2} \omega^{2}[r r]_{m}^{(2)}+[p p]_{m}^{(2)}\right)$, where $[r r]_{m}^{(2)}$ means coupled to angular momentum rank 2 and projection $m$, and $\mathcal{N}_{N R}=\frac{1}{2 \sqrt{2} \hbar M \omega}\left(M^{2} \omega^{2} r^{2}+p^{2}\right)-\frac{3}{2}$. They form the closed $\mathrm{U}(3)$ algebra

$$
\begin{gathered}
{\left[\mathcal{N}_{N R}, \vec{\ell}\right]=\left[\mathcal{N}_{N R}, q\right]=0,} \\
{[\vec{\ell}, \vec{\ell}]^{(t)}=-\sqrt{2} \vec{\ell} \delta_{t, 1},[\vec{\ell}, q]^{(t)}=-\sqrt{6} q \delta_{t, 2},[q, q]^{(t)}=3 \sqrt{10} \vec{\ell} \delta_{t, 1},}
\end{gathered}
$$

with $\mathcal{N}_{N R}$ generating a $\mathrm{U}(1)$ algebra whose eigenvalues are the total number of quanta $N$ and $\vec{\ell}, q$ generating an $\mathrm{SU}(3)$ algebra. In the above we use the coupled commutation relation between two tenors, $T_{1}^{\left(t_{1}\right)}, T_{2}^{\left(t_{2}\right)}$ of rank $t_{1}, t_{2}$ which is $\left[T_{1}^{\left(t_{1}\right)}, T_{2}^{\left(t_{2}\right)}\right]^{(t)}=\left[T_{1}^{\left(t_{1}\right)} T_{2}^{\left(t_{2}\right)}\right]^{(t)}-$ $(-1)^{t_{1}+t_{2}-t}\left[T_{2}^{\left(t_{2}\right)} T_{1}^{\left(t_{1}\right)}\right]^{(t)}[8]$.

The relativistic orbital angular momentum generators $\vec{L}$ are given in Eq. (2). We shall now determine the the quadrupole operator $Q_{m}$ and monopole operator $\mathcal{N}$ that commute with the Hamiltonian in Eq.(1). In order for the quadrupole generator

$$
Q_{m}=\left(\begin{array}{cc}
\left(Q_{m}\right)_{11} & \left(Q_{m}\right)_{12} \vec{\sigma} \cdot \vec{p} \\
\vec{\sigma} \cdot \vec{p}\left(Q_{m}\right)_{21} & \vec{\sigma} \cdot \vec{p}\left(Q_{m}\right)_{22} \vec{\sigma} \cdot \vec{p}
\end{array}\right),
$$


to commute with the Hamiltonian, $\left[Q_{m}, H\right]=0$, the matrix elements must satisfy the con 4 ditions,

$$
\begin{gathered}
\left(Q_{m}\right)_{12}=\left(Q_{m}\right)_{21}, \\
{\left[\left(Q_{m}\right)_{11}, V\right]+\left[\left(Q_{m}\right)_{12}, p^{2}\right]=0,} \\
{\left[\left(Q_{m}\right)_{12}, V\right]+\left[\left(Q_{m}\right)_{22}, p^{2}\right]=0,} \\
\left(Q_{m}\right)_{11}=\left(Q_{m}\right)_{12}(V+2 M)+\left(Q_{m}\right)_{22} p^{2} .
\end{gathered}
$$

One solution is

$$
Q_{m}=\lambda_{2}\left(\begin{array}{cc}
\frac{M \omega^{2}}{2}\left(\frac{M \omega^{2}}{2} r^{2}+2 M\right)[r r]_{m}^{(2)}+[p p]_{m}^{(2)} & \frac{M \omega^{2}}{2}[r r]_{m}^{(2)} \vec{\sigma} \cdot \vec{p} \\
\vec{\sigma} \cdot \vec{p} \frac{M \omega^{2}}{2}[r r]_{m}^{(2)} & {[p p]_{m}^{(2)}}
\end{array}\right),
$$

where $\lambda_{2}$ is an overall constant undetermined by the commutation of $Q_{m}$ with the Dirac Hamiltonian.

For this quadrupole operator to form a closed algebra, the commutation with itself must be the orbital angular momentum operator as in Eq. (3). This commutation relation gives

$$
[Q, Q]^{(t)}=\sqrt{10} \lambda_{2}^{2} M \omega^{2} \hbar^{2}\left(\begin{array}{cc}
\left(\frac{M \omega^{2}}{2} r^{2}+2 M\right) \vec{\ell} & \vec{\ell} \vec{\sigma} \cdot \vec{p} \\
\vec{\sigma} \cdot \vec{p} \vec{\ell} & 0
\end{array}\right)=\sqrt{10} \lambda_{2}^{2} M \omega^{2} \hbar^{2}(H+M) \vec{L} \delta_{t, 1},
$$

and we get the desired result if $\lambda_{2}=\sqrt{\frac{3}{M \omega^{2} \hbar^{2}(H+M)}}$. The quadrupole operator then becomes 
$Q_{m}=\sqrt{\frac{3}{M \omega^{2} \hbar^{2}(H+M)}}\left(\begin{array}{cc}\frac{M \omega^{2}}{2}\left(\frac{M \omega^{2}}{2} r^{2}+2 M\right)[r r]_{m}^{(2)}+[p p]_{m}^{(2)} & \frac{M \omega^{2}}{2}[r r]_{m}^{(2)} \vec{\sigma} \cdot \vec{p} \\ \vec{\sigma} \cdot \vec{p} \frac{M \omega^{2}}{2}[r r]_{m}^{(2)} & {[p p]_{m}^{(2)}}\end{array}\right)$

In order for the monopole generator

$$
\mathcal{N}=\left(\begin{array}{cc}
(\mathcal{N})_{11} & (\mathcal{N})_{12} \vec{\sigma} \cdot \vec{p} \\
\vec{\sigma} \cdot \vec{p}(\mathcal{N})_{21} & \vec{\sigma} \cdot \vec{p}(\mathcal{N})_{22} \vec{\sigma} \cdot \vec{p}
\end{array}\right)+\mathcal{N}_{0}
$$

to commute with the Hamiltonian, $[\mathcal{N}, H]=0$, the matrix elements must satisfy the conditions in Eq.(15) with $Q_{m}$ replaced by $\mathcal{N} \cdot \mathcal{N}_{0}$ is a constant. A solution is

$$
\mathcal{N}=\lambda_{0}\left(\begin{array}{cc}
\frac{M \omega^{2}}{2}\left(\frac{M \omega^{2}}{2} r^{2}+2 M\right) r^{2}+p^{2} & \frac{M \omega^{2}}{2} r^{2} \vec{\sigma} \cdot \vec{p} \\
\vec{\sigma} \cdot \vec{p} \frac{M \omega^{2}}{2} r^{2} & p^{2}
\end{array}\right)+\mathcal{N}_{0}
$$

Straightfoward calculations show that $\mathcal{N}$ commutes with the the other generators as well as the Dirac Hamiltonian and consequently is the $\mathrm{U}(1)$ generator. However, the constants $\lambda_{0}, \mathcal{N}_{0}$ are undetermined by these commutation relations. These constants are determined instead by requiring that the eigenvalue of $\mathcal{N}$ is the the total harmonic oscillator number, $N$; that is, $\mathcal{N} \Psi_{N}=N \Psi_{N}$, where $\Psi_{N}$ are the eigenfunctions of the Dirac Hamiltonian, $H \Psi_{N}=E_{N} \Psi_{N}$. Using the facts that [4, 5]

$$
\Psi_{N}=\left(\begin{array}{c}
g \\
\frac{\vec{\sigma} \cdot \vec{p}}{E_{N}+M}
\end{array}\right),\left[p^{2}+\left(E_{N}+M\right) V(r)-2 \hbar \sqrt{\left(E_{N}+M\right) M \omega^{2}}\left(N+\frac{3}{2}\right)\right] g=0,
$$

we derive that

$$
\mathcal{N} \Psi_{N}=\left[2 \hbar \lambda_{0} \sqrt{\left(E_{N}+M\right) M \omega^{2}}\left(N+\frac{3}{2}\right)+\mathcal{N}_{0}\right] \Psi_{N}=N \Psi_{N}
$$

which determines $\lambda_{0}=\frac{1}{2 \hbar \sqrt{(H+M) M \omega^{2}}}, \mathcal{N}_{0}=-\frac{3}{2}$. 
In the non-relativisitc limit, $H \rightarrow M, M \rightarrow \infty$,

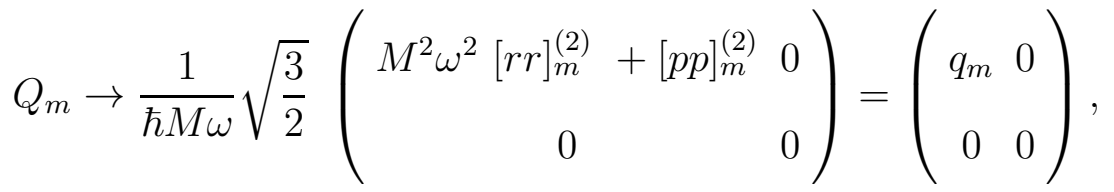

$$
\begin{aligned}
& \mathcal{N} \rightarrow \frac{1}{2 \sqrt{2} \hbar M \omega}\left(\begin{array}{rr}
M^{2} \omega^{2} r^{2}+p^{2} & 0 \\
0 & 0
\end{array}\right)-\frac{3}{2}=\left(\begin{array}{cc}
\mathcal{N}_{N R} & 0 \\
0 & -\frac{3}{2}
\end{array}\right)
\end{aligned}
$$

which agrees with the non-relativistic generators.

The commutation relations are then those of the $\mathrm{U}(3)$ algebra,

$$
\begin{gathered}
{[\mathcal{N}, \vec{L}]=\left[\mathcal{N}, Q_{m}\right]=0 .} \\
{[\vec{L}, \vec{L}]^{(t)}=-\sqrt{2} \vec{L} \delta_{t, 1},[\vec{L}, Q]^{(t)}=-\sqrt{6} Q \delta_{t, 2},[Q, Q]^{(t)}=3 \sqrt{10} \vec{L} \delta_{t, 1} .}
\end{gathered}
$$

The spin generators in Eq.(2), $\vec{S}$, commute with the U(3) generators as well as the Dirac Hamiltonian, and so the invariance group is $\mathrm{U}(3) \times \mathrm{SU}(2)$, where the $\mathrm{SU}(2)$ is generated by the spin generators, $[\vec{S}, \vec{S}]^{(t)}=-\sqrt{2} \vec{S} \delta_{t, 1}$.

The Dirac Hamiltonian for a spherical harmonic oscillator with pseudospin symmetry is [9]

$$
\tilde{H}=\vec{\alpha} \cdot \vec{p}+\beta M+(1-\beta) V(r),
$$

which explains the pseudospin doublets observed in nuclei [4]. This pseudospin Hamiltonian can be obtained from the spin Hamiltonian with a transformation by $\gamma_{5}=\left(\begin{array}{ll}0 & 1 \\ 1 & 0\end{array}\right)$ and $M \rightarrow-M$, but $M \omega^{2} \rightarrow M \omega^{2}$. The generators for the pseudospin [10] and pseudo- $\mathrm{U}(3)$ algebra which commute with the Dirac Hamiltonian, $[\tilde{H}, \overrightarrow{\tilde{S}}]=[\tilde{H}, \overrightarrow{\tilde{L}}]=\left[\tilde{H}, \tilde{Q}_{m}\right]=[\tilde{H}, \tilde{\mathcal{N}}]=$ 
0 , are then obtained by the same transformation and are given by

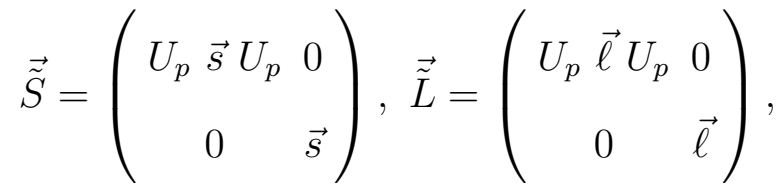

$$
\begin{aligned}
& \tilde{Q}_{m}=\sqrt{\frac{3}{M \omega^{2} \hbar^{2}(\tilde{H}-M)}}\left(\begin{array}{cc}
{[p p]_{m}^{(2)}} & \vec{\sigma} \cdot \vec{p} \frac{M \omega^{2}}{2}[r r]_{m}^{(2)} \\
\frac{M \omega^{2}}{2}[r r]_{m}^{(2)} \vec{\sigma} \cdot \vec{p} & \frac{M \omega^{2}}{2}\left(\frac{M \omega^{2}}{2} r^{2}-2 M\right)[r r]_{m}^{(2)}+[p p]_{m}^{(2)}
\end{array}\right), \\
& \tilde{\mathcal{N}}=\frac{1}{2 \hbar \sqrt{M \omega^{2}(\tilde{H}-M)}}\left(\begin{array}{cc}
p^{2} & \vec{\sigma} \cdot \vec{p} \frac{M \omega^{2}}{2} r^{2} \\
\frac{M \omega^{2}}{2} \vec{\sigma} \cdot \vec{p} r^{2} & \frac{M \omega^{2}}{2}\left(\frac{M \omega^{2}}{2} r^{2}-2 M\right) r^{2}+p^{2}
\end{array}\right)-\frac{3}{2} .
\end{aligned}
$$

The relativistic non-spherical harmonic oscillator has also been solved analytically [5] as well. The relativistic axially symmetric deformed harmonic oscillator will have a U(2) symmetry as does the non-relativistic axially symmetric deformed harmonic oscillator. This will be discussed in a forthcoming paper.

In summary, we have shown that a Dirac Hamiltonian with equal scalar and vector harmonic oscillator potentials has an $\mathrm{U}(3) \times \mathrm{SU}(2)$ symmetry and that a Dirac Hamiltonian with scalar and vector harmonic oscillator potentials equal in magnitude but opposite in sign has a pseudo- $\mathrm{U}(3) \times$ pseudo-SU(2) symmetry and we have derived the corresponding generators for each case. If speculation that an anti-nucleon can be bound inside a nucleus is valid 11], the anti-nucleon spectrum will have an approximate spin symmetry and, most likely an approximate $\mathrm{U}(3)$ symmetry, because the vector and scalar potentials are approximately equal and are very strong [4].

[1] J.P. Elliott, Proc. Roy. Soc. A 245 , 128 (1958). 
[2] I. Talmi, Simple Models of Complex Nuclei, (Hardwood, Switzerland, 1993).

[3] See for example, Extended Density Functionals in Nuclear Structure Physics, edited by G. Lalazissis, P. Ring, and D. Vretenar, Lecture Notes in Physics Vol 641 (SpringerVerlag,Berlin,2004), p. 219.

[4] J. N. Ginocchio, Physics Reports 414, 165 (2005).

[5] J. N. Ginocchio, Phys. Rev. C 69, 034318 (2004).

[6] J. S. Bell and H. Ruegg, Nucl. Phys. B 98, 151 (1975).

[7] A. L. Blokhin, C. Bahri, and J. P. Draayer, Phys. Rev. Lett. 74, 4149 (1995).

[8] J. B. French, in Many-Body Description of Nuclear Structure and Reactions, edited by C. Bloch, (Academic Press, New York, 1966).

[9] J. N. Ginocchio, Phys. Rev. Lett. 78, 436 (1997).

[10] J. N. Ginocchio and A. Leviatan, Phys. Lett. B 425, 1 (1998).

[11] T. Bürvenich et al, Phys. Lett. B 542, 261 (2002). 